Proyecciones Journal of Mathematics

Vol. 30, No 2, pp. 265-275, August 2011.

Universidad Católica del Norte

Antofagasta - Chile

\title{
An extension of Sheffer polynomials
}

\author{
A. K. SHUKLA \\ S. V. NATIONAL INSTITUTE OF TECHNOLOGY, INDIA \\ S. J. RAPELI \\ S. V. NATIONAL INSTITUTE OF TECHNOLOGY, INDIA \\ Received : February 2011. Accepted : September 2011
}

\begin{abstract}
Sheffer [Some properties of polynomial sets of type zero, Duke Math. J. 5 (1939), pp.590-622] studied polynomial sets zero type and many authors investigated various properties and its applications. In the sequel to the study of Sheffer Polynomials, an attempt is made to generalize the Sheffer polynomials by using partial differential operator.
\end{abstract}

Keywords : Appell sets, Differential operator, Sheffer polynomials, generalized Sheffer polynomials.

AMS Subject Classification (2010): 33C65, 33F99, 44A45,46G25. 


\section{Introduction}

Sheffer [8] wrote remarkable paper on some properties of polynomials of type zero, Huff and Rainville [4] obtained generating function of Sheffer Polynomial and Rainville [7] reported this work in his book. Al Salam and Verma [1] studied the generalized Sheffer polynomials and Bahman Alidad [2] studied Sheffer polynomials in two variables.Recently Galiffa Joseph [3] worked on Sheffer B-type 1 orthogonal polynomial sequences in his thesis.

A polynomial set $\left\{P_{n}\right\}_{n \geq 0}$ is said to be of Sheffer A-type zero [5] if and only if it has a generating function of the form

$$
A(t) \exp (x G(t))=\sum_{n=0}^{\infty} P_{n}(x) t^{n},
$$

where $A(t)$ and $G(t)$ are two formal power series

$$
A(t)=\sum_{k=0}^{\infty} a_{k} t^{k}, \quad a_{0} \neq 0, \quad G(t)=\sum_{k=0}^{\infty} g_{k} t^{k+1}, \quad g_{0} \neq 0 .
$$

This has the property: $J(D) P_{0}(x)=0$ and $J P_{n}(x)=P_{n-1}(x), \quad n \geq 1$; where $J(D)$ is defined by

$$
J=J(D)=\sum_{k=0}^{\infty} a_{k} D^{k+1}, \quad a_{0} \neq 0 \quad \text { and } \quad D=\frac{d}{d x} .
$$

Bahman Alidad [2] studied polynomials in two variables of Sheffer A-type zero and also proved some related theorems. Sheffer [8] and Steffensen [9] generalized Appell sets by considering a linear differential operator of infinite order with constant coefficients

$$
L(D) P_{n}(x)=P_{n-1}(x), \quad \text { where } L(D)=\sum_{k=0}^{\infty} c_{k} D^{k+1}, \quad c_{0} \neq 0 .
$$

Osegove [6] has also generalized Appell sets and studied polynomial sets which has the property $D^{r} P_{n}(x)=P_{n-r}(x), \quad(n=r, r+1, \ldots)$, where $r$ is a fixed positive number.

Al Salam and Verma [1] generalized Sheffer- Steffensen polynomials and defined as

$J(D) P_{n}(x)=P_{n-r}(x), \quad n=r, r+1, \ldots, \quad$ where $J(D)=\sum_{k=0}^{\infty} a_{k} D^{k+r}, a_{0} \neq 0$ 
and $a_{k}(k \geq 0)$ is independent of $x$.

In sequel to the study, we consider a class of polynomials that contain both the Sheffer-Steffensen sets and the Osegove sets of polynomials in two variables.

Let $\left\{P_{n}(x, y)\right\}$ be a simple set polynomials in two variables $x$ and $y$, i.e. a sequence of polynomials $P_{0}(x, y), P_{1}(x, y), P_{2}(x, y), \ldots$ for which the degree of $P_{n}(x, y)$ is exactly $n$.

A polynomial set in two variables is said to be Appell set if $P_{n}^{\prime}(x, y)=$ $P_{n-1}(x, y)$, for all $n \geq 1$. A well known characteristic property of Appell set is the existence of a (formal) power series $A(t)=\sum_{n=0}^{\infty} a_{n} t^{n}, \quad a_{0} \neq 0$ such that

$$
\sum_{n=0}^{\infty} P_{n}(x, y) t^{n}=A(t) \exp (x t) \exp (y t)
$$

In generalized Appell sets, consider a linear differential operator of infinite order with constant coefficients

$$
L(D)=\sum_{k=0}^{\infty} C_{k} D^{k+1}, \quad C_{0} \neq 0,
$$

as a generalization of the differential operator $D=\frac{\partial}{\partial x}+\frac{\partial}{\partial y}$.

The polynomial set which satisfy the following property

$$
L(D) P_{n}(x, y)=P_{n-1}(x, y),
$$

Bahman Alidad [2] proved that polynomial sets have the property as Equation (1.3) if and only if polynomial sets have the generating function in following form

$$
A(t) \exp (x G(t)) \exp (y H(t))=\sum_{n=0}^{\infty} P_{n}(x, y) t^{n}
$$

where

$$
G(t)=\sum_{n=0}^{\infty} g_{n} t^{n+1}, \quad g_{0} \neq 0
$$




$$
\begin{aligned}
& H(t)=\sum_{n=0}^{\infty} h_{n} t^{n+1}, \quad h_{0} \neq 0, \\
& A(t)=\sum_{n=0}^{\infty} a_{n} t^{n}, \quad a_{0} \neq 0 .
\end{aligned}
$$

The polynomial sets in two variables have the property as given below

$$
D^{r} P_{n}(x, y)=P_{n-r}(x, y) \quad(n=r, r+1, \ldots),
$$

where $r$ is a (fixed) positive integer and $D=\frac{\partial}{\partial x}+\frac{\partial}{\partial y}$.

\section{The generalized Sheffer-Steffensen polynomials}

Let $r$ be a fixed positive integer and we consider the differential operator (formal) of infinite order

$$
J(D)=\sum_{k=0}^{\infty} a_{k} D^{k+r}, \quad a_{0} \neq 0, \quad D=\frac{\partial}{\partial x}+\frac{\partial}{\partial y},
$$

where $a_{k}(k \geq 0)$ is independent of $x$. We shall say that the polynomial set $\left\{P_{n}(x, y)\right\}$ is in $S^{(r)}$ if there is an operator $J(D)$ of the form Equation (1.6) such that

$$
J(D) P_{n}(x, y)=P_{n-r}(x, y) \quad(n=r, r+1, r+2, \ldots)
$$

Obviously $S^{(r)}$ contains the Osegove class $A^{(r)}$ of polynomial sets which satisfy Equation (1.5). We clearly see that $S^{(1)}$ is the class of Sheffer Atype zero polynomials. It is also clear that $S^{(r)}$ may be partitioned into equivalence classes $\left\{S_{J}^{(r)}\right\}$ where $S_{J}^{(r)}$ is the class of all polynomial sets with the property that

$$
J(D) P_{n}(x, y)=P_{n-r}(x, y),
$$

for a fixed operator $J$. $S_{J}^{(r)}$

If $\left\{p_{n}(x, y)\right\} \in S_{J}^{(r)},\left\{q_{n}(x, y)\right\} \in S_{J}^{(r)}$ then $\left\{\alpha p_{n}(x, y)+\beta q_{n}(x, y)\right\} \in$ where $\alpha, \beta$ are constants or are periodic in $n$ of period $r$.

Theorem 2.1: Let $\left\{P_{n}(x, y)\right\}$ be a polynomial set in two variables. In order to that $\left\{P_{n}(x, y)\right\}$ be in $S^{(r)}$, it is necessary and sufficient that there exist (formal) power series

$$
G(t)=\sum_{i=1}^{\infty} g_{i} t^{i}, \quad g_{1} \neq 0,
$$




$$
H(t)=\sum_{i=1}^{\infty} h_{i} t^{i}, \quad h_{1} \neq 0
$$

$$
A_{s}(t)=\sum_{i=0}^{\infty} \alpha_{i}^{(s)} t^{i}, \quad\left(\text { not all } \alpha_{0}^{(\mathrm{s})} \text { are zeros }\right)
$$

such that

$$
\sum_{i=1}^{r} A_{i}(t) \exp \left(\left(x G ( \varepsilon _ { i } ( t ) ) \operatorname { e x p } \left(\left(y H\left(\varepsilon_{i}(t)\right)=\sum_{n=0}^{\infty} P_{n}(x, y) t^{n}\right.\right.\right.\right.
$$

Proof: If $F(t)=\sum_{k=0}^{\infty} \alpha_{k} t^{k}, \alpha_{0} \neq 0$, is a formal power series then we can find another formal power series $f(t)=\sum_{k=0}^{\infty} \beta_{k} t^{k}, \beta_{0} \neq 0$ such that

$$
F(t)=(f(t))^{k}, \quad k=1,2, \ldots .
$$

Now let the polynomial sets $\left\{P_{n}(x, y)\right\} \in S^{(r)}$ and suppose that the differential operator corresponding to $\left\{P_{n}(x, y)\right\}$ is

$$
J(D)=\sum_{k=0}^{\infty} a_{k} D^{k+r}, \quad a_{0} \neq 0, \quad D=\frac{\partial}{\partial x}+\frac{\partial}{\partial y} .
$$

We write $J(t)=t^{r} F(t)$ and using Equation (1.12)

$$
J(t)=\{t f(t)\}^{r}=\{L(t)\}^{r},
$$

therefore, that $L(D)$ is a differential operator of the form Equation (1.2).

Let

$$
F(x, y, t)=\sum_{n=0}^{\infty} P_{n}(x, y) t^{n}
$$

Now

$$
\begin{gathered}
J(D) F(x, y, t)=\sum_{n=0}^{\infty} J(D) P_{n}(x, y) t^{n} \\
=\sum_{n=r}^{\infty} P_{n-r}(x, y) t^{n} \\
=\sum_{n=0}^{\infty} P_{n}(x, y) t^{n+r}
\end{gathered}
$$




$$
\begin{gathered}
=t^{r} \sum_{n=0}^{\infty} P_{n}(x, y) t^{n} \\
J(D) F(x, y, t)=t^{r} F(x, y, t) \\
\left\{J(D)-t^{r}\right\} F(x, y, t)=0
\end{gathered}
$$

Consequently if $\varepsilon_{1}, \varepsilon_{2}, \ldots, \varepsilon_{r}$ are the $r$ roots of unity, we get that

$$
\left\{L(D)-\varepsilon_{i} t\right\} F(x, y, t)=0 \quad(i=1,2, \ldots, r) .
$$

Hence

$$
F(x, y, t)=\sum_{i=1}^{r} A_{i}(t) \exp \left(x G\left(\varepsilon_{i} t\right)\right) \exp \left(y H\left(\varepsilon_{i} t\right)\right),
$$

where $L(H(t))=t$.

Conversely if $F(x, y, t)$ has the form as Equation (1.13), then it follows that

$$
\begin{aligned}
J(D) F(x, y, t) & =t^{r} F(x, y, t) \\
J(D) \sum_{n=0}^{\infty} P_{n}(x, y) t^{n} & =t^{r} \sum_{n=0}^{\infty} P_{n}(x, y) t^{n} \\
\sum_{n=0}^{\infty} J(D) P_{n}(x, y) t^{n} & =\sum_{n=0}^{\infty} P_{n}(x, y) t^{n+r} \\
\sum_{n=r}^{\infty} J(D) P_{n}(x, y) t^{n} & =\sum_{n=r}^{\infty} P_{n-r}(x, y) t^{n}
\end{aligned}
$$

So that

$$
J(D) P_{n}(x, y)=P_{n-r}(x, y) .
$$

This completes the proof of Theorem 2.1.

Theorem 2.2: Let two polynomial sequences $P_{n}(x, y)$ and $Q_{n}(x, y)$ are in $S^{(r)}$ and there is an arbitrary sequence of numbers $\left\{a_{n}\right\}, a_{0} \neq 0$, if and only if

$$
Q_{n}(x, y)=\sum_{k=0}^{n} a_{n-k} P_{k}(x, y)
$$


Proof: Consider $\left\{P_{n}(x, y)\right\},\left\{Q_{n}(x, y)\right\} \in S^{(r)}$.

Therefore $P_{n}(x, y)$ and $Q_{n}(x, y)$ belong to the same operator $J(D)$ as in form of Equation (1.6) such that

$$
J(D) P_{n}(x, y)=P_{n-r}(x, y) \text { and } J(D) Q_{n}(x, y)=Q_{n-r}(x, y),
$$

where $(n=r, r+1, r+2, \ldots)$.

Since $P_{n}(x, y)$ and $Q_{n}(x, y)$ are simple set of polynomials [5] in two variables,

$$
Q_{n}(x, y)=\sum_{k=0}^{n} a_{n, k} P_{k}(x, y)
$$

Thus

$$
\begin{aligned}
\sum_{k=0}^{n-r} a_{n-r, k} P_{k} & (x, y)=Q_{n-r}(x, y)=J Q_{n}(x, y) \\
= & \sum_{k=r}^{n} a_{n, k} J P_{k}(x, y) \\
= & \sum_{k=r}^{n} a_{n, k} P_{k-r}(x, y) \\
& =\sum_{k=0}^{n-r} a_{n, k+r} P_{k}(x, y)
\end{aligned}
$$

Hence $a_{n-r, k}=a_{n, k+r} \quad$ or $\quad a_{n, k+r}=a_{n-k-r, 0}$, yields Equation (1.14).

Conversely: Assume Equation (1.14) to hold. There exists an operator $J$ to which $P_{n}(x, y)$ belongs. It follows from Equation (1.14) that $Q_{n}(x, y)$ also belongs to same operator $J$. This proves Theorem 2.2 .

Theorem 2.3: Let $P_{n}(x, y)$ be of Sheffer A-type zero, $P_{n}(x, y)$ belong to the operator $J(D)$ and have the generating function in equation (1.11) of Theorem 2.1. There exist sequences $\alpha_{k}^{(s)}, \mu_{k}$ and $\eta_{k}$, independent of $x, y$ and $n$, such that for all $n \geq 1$,

$$
n P_{n}(x, y)=\sum_{i=1}^{r} S_{i, n}(x, y) \psi\left(\varepsilon_{i} t\right)
$$

where $S_{i, n}(x, y)=\alpha_{n-1}^{(i)}+x \varepsilon_{i}^{n} \mu_{n-1}+y \varepsilon_{i}^{n} \eta_{n-1}$ and $\psi\left(\varepsilon_{i} t\right)=A_{i}(t) \exp \left(x G\left(\varepsilon_{i} t\right)\right) \exp \left(y H\left(\varepsilon_{i} t\right)\right)$ and $\mu_{k}=(k+1) g_{k}$ in terms of $g_{k}$ of Equation (1.8) and $\eta_{k}=(k+1) h_{k}$, in terms of $h_{k}$ of Equation (1.9). 
Proof: Consider the series

$$
\sum_{n=0}^{\infty} P_{n}(x, y) t^{n}=\sum_{i=1}^{r} A_{i}(t) \exp \left(x G\left(\varepsilon_{i} t\right)\right) \exp \left(y H\left(\varepsilon_{i} t\right)\right)
$$

and writing this in the following form

$$
t \frac{\partial}{\partial t}\left(\sum_{n=0}^{\infty} P_{n}(x, y) t^{n}\right)=t \frac{\partial}{\partial t}\left(\sum_{i=1}^{r} A_{i}(t) \exp \left(x G\left(\varepsilon_{i} t\right)\right) \exp \left(y H\left(\varepsilon_{i} t\right)\right)\right)
$$

or

$$
\sum_{n=0}^{\infty} n P_{n}(x, y) t^{n}=t\left[\begin{array}{c}
\sum_{i=1}^{r}\left\{A_{i}^{\prime}(t)+x \varepsilon_{i} G^{\prime}\left(\varepsilon_{i} t\right) A_{i}(t)+y \varepsilon_{i} H^{\prime}\left(\varepsilon_{i} t\right) A_{i}(t)\right\} \\
\exp \left(x G\left(\varepsilon_{i} t\right)\right) \exp \left(y H\left(\varepsilon_{i} t\right)\right)
\end{array}\right]
$$

On simplifying above equation, yields

$$
\begin{aligned}
& \sum_{n=0}^{\infty} n P_{n}(x, y) t^{n}=t\left[\begin{array}{c}
\left.\sum_{i=1}^{r}\left\{\frac{A_{i}^{\prime}(t)}{A_{i}(t)}+x \varepsilon_{i} G^{\prime}\left(\varepsilon_{i} t\right)+y \varepsilon_{i} H^{\prime}\left(\varepsilon_{i} t\right)\right\} A_{i}(t)\right] \\
\exp \left(x G\left(\varepsilon_{i} t\right)\right) \exp \left(y H\left(\varepsilon_{i} t\right)\right)
\end{array}\right] \\
= & t\left[\sum_{i=1}^{r}\left\{\sum_{n=0}^{\infty} \alpha_{n}^{(i)} t^{n}+x \varepsilon_{i} \sum_{n=0}^{\infty} \mu_{n} \varepsilon_{i}^{n} t^{n}+y \varepsilon_{i} \sum_{n=0}^{\infty} \eta_{n} \varepsilon_{i}^{n} t^{n}\right\} A_{i}(t)\right] \\
= & t\left[\sum_{i=1}^{r}\left\{\sum_{n=0}^{\infty}\left(\alpha_{n}^{(i)}+x \varepsilon_{i}^{n+1} \mu_{n}+y \varepsilon_{i}^{n+1} \eta_{n}\right) t^{n}\right\} A_{i}(t) \exp \left(x G\left(\varepsilon_{i} t\right)\right)\right] \\
= & \sum_{n=0}^{\infty} \sum_{i=1}^{r}\left(\alpha_{n}^{(i)}+x \varepsilon_{i}^{n+1} \mu_{n}+y \varepsilon_{i}^{n+1} \eta_{n}\right) A_{i}(t) \exp \left(x G\left(\varepsilon_{i} t\right)\right) \exp \left(y H\left(\varepsilon_{i} t\right)\right) t^{n+1} \\
= & \sum_{n=1}^{\infty} \sum_{i=1}^{r}\left(\alpha_{n-1}^{(i)}+x \varepsilon_{i}^{n} \mu_{n-1}+y \varepsilon_{i}^{n} \eta_{n-1}\right) A_{i}(t) \exp \left(x G\left(\varepsilon_{i} t\right)\right) \exp \left(y H\left(\varepsilon_{i} t\right)\right) t^{n}
\end{aligned}
$$

So that

$$
\sum_{n=1}^{\infty} n P_{n}(x, y) t^{n}=\sum_{n=1}^{\infty} \sum_{i=1}^{r} S_{i, n}(x, y) \quad \psi\left(\varepsilon_{i} t\right) t^{n}
$$

Hence

$$
n P_{n}(x, y)=\sum_{i=1}^{r} S_{i, n}(x, y) \quad \psi\left(\varepsilon_{i} t\right)
$$

where

$$
S_{i, n}(x, y)=\alpha_{n-1}^{(i)}+x \varepsilon_{i}^{n} \mu_{n-1}+y \varepsilon_{i}^{n} \eta_{n-1}
$$


and

$$
\psi\left(\varepsilon_{i} t\right)=A_{i}(t) \exp \left(x G\left(\varepsilon_{i} t\right)\right) \exp \left(y H\left(\varepsilon_{i} t\right)\right) .
$$

This gives the statement.

Theorem 2.4: A necessary and sufficient condition that $P_{n}(x, y)$ be of Sheffer A-type zero, there exists sequence $g_{k}$ and $h_{k}$, independent of $x, y$ and $n$, such that

$$
\left(\frac{\partial}{\partial x}+\frac{\partial}{\partial y}\right) P_{n}(x, y)=\sum_{i=1}^{r}\left(\varepsilon_{i}^{n} g_{n-1}+\varepsilon_{i}^{n} h_{n-1}\right) \psi\left(\varepsilon_{i} t\right)
$$

Proof:

$$
\begin{gathered}
\left(\frac{\partial}{\partial x}+\frac{\partial}{\partial y}\right)\left(\sum_{n=0}^{\infty} P_{n}(x, y) t^{n}\right)=\left(\frac{\partial}{\partial x}+\frac{\partial}{\partial y}\right)\left[\begin{array}{c}
\sum_{i=1}^{r} A_{i}(t) \exp \left(x G\left(\varepsilon_{i} t\right)\right) \\
\exp \left(y H\left(\varepsilon_{i} t\right)\right)
\end{array}\right] \\
\sum_{n=0}^{\infty}\left(\frac{\partial}{\partial x}+\frac{\partial}{\partial y}\right) P_{n}(x, y) t^{n}=\sum_{i=1}^{r}\left\{G\left(\varepsilon_{i} t\right)+H\left(\varepsilon_{i} t\right)\right\} A_{i}(t) \exp \left(x G\left(\varepsilon_{i} t\right)\right) \\
\exp \left(y H\left(\varepsilon_{i} t\right)\right) \\
=\sum_{i=1}^{r} \sum_{n=0}^{\infty}\left(\varepsilon_{i}^{n+1} g_{n}+\varepsilon_{i}^{n+1} h_{n}\right) t^{n+1} \psi\left(\varepsilon_{i} t\right) \\
=\sum_{n=1}^{\infty} \sum_{i=1}^{r}\left(\varepsilon_{i}^{n} g_{n-1}+\varepsilon_{i}^{n} h_{n-1}\right) \psi\left(\varepsilon_{i} t\right) t^{n}
\end{gathered}
$$

Thus

$$
\left(\frac{\partial}{\partial x}+\frac{\partial}{\partial y}\right) P_{n}(x, y)=\sum_{i=1}^{r}\left(\varepsilon_{i}^{n} g_{n-1}+\varepsilon_{i}^{n} h_{n-1}\right) \psi\left(\varepsilon_{i} t\right)
$$

where

$$
\psi\left(\varepsilon_{i} t\right)=A_{i}(t) \exp \left(x G\left(\varepsilon_{i} t\right)\right) \exp \left(y H\left(\varepsilon_{i} t\right)\right) .
$$

Hence, we get theorem 2.4 . 


\section{References}

[1] W. A. Al Salam and A. Verma, Generalized Sheffer Polynomials, Duke Math. J. 37, pp. 361-365, (1970).

[2] B. Alidad, On some problems of special functions and structural matrix analysis, Ph.D. diss., Aligarh Muslim University, (2008).

[3] Joseph D. Galiffa, The Sheffer B-type 1 orthogonal polynomial sequences, Ph.D. diss., University of Central Florida, (2009).

[4] William N. Huff and E. D. Rainville, On the Sheffer A-type of polynomials generated by $\phi(t) f(x t)$, Proc. Amer. Math. Soc. 3, pp. 296-299 (1952).

[5] E. B. Mc Bride, Obtaining Generating Functions, Springer, New York, (1971).

[6] V. B. Osegove, Some extremal properties of generalized Appell polynomials, Soviet Math. Five, pp. 1651-1653, (1964).

[7] E. D. Rainville, Special Functions, the Macmillan Company, New York, (1960).

[8] I. M. Sheffer, Some properties of polynomial sets of type zero, Duke Math. J. 5, pp. 590-622, (1939).

[9] J. F. Steffensen, The Poweroid, an extension of the mathematical notion of power, Acta Math. 73, pp. 333-366, (1941).

\section{A. K. Shukla}

Department of Mathematics

S. V. National Institute of Technology

Surat-395 007

India

e-mail : ajayshukla2@rediffmail.com,

and 
S. J. Rapeli

Department of Mathematics

S. V. National Institute of Technology

Surat-395 007

India

e-mail : shrinu0711@gmail.com 